\title{
Determining the adsorption-induced surface stress and mass by measuring the shifts of resonant frequencies
}

\author{
Yin Zhang* \\ State Key Laboratory of Nonlinear Mechanics (LNM), Institute of Mechanics, Chinese Academy of Sciences, Beijing 100190, China
}

\section{A R T I C L E I N F O}

\section{Article history:}

Received 9 August 2012

Received in revised form

20 November 2012

Accepted 15 January 2013

Available online 1 February 2013

\section{Keywords:}

Surface stress

Resonant frequencies

Adsorption

Resonator sensor

\begin{abstract}
A B S T R A C T
The adsorption-induced surface stress and mass can cause the resonant frequency shifts of a microcantilever, which is used as the sensing mechanism for a resonator sensor. Determining the adsorption-induced surface stress and mass from the experimentally measured data of resonant frequencies forms an inverse problem. Because there are infinite combinations of surface stress and mass which can result in the same change of one resonant frequency, the previous studies usually measure surface stress or mass by one measurement method and then find the other by another different measurement method. This study shows that surface stress and mass have different impacts on the resonant frequencies of a microcantilever. Two resonant frequencies are used to uniquely determine the adsorption-induced surface stress and mass. Mathematically, the new method presented in this study provides an efficient and straightforward solution to the inverse problem and its accuracy is also demonstrated. Physically, the new method only requires the dynamic mode to measure the resonant frequencies, which should be of a great help to various sensor applications.
\end{abstract}

(c) 2013 Elsevier B.V. All rights reserved.

\section{Introduction}

Microcantilever sensor has become increasingly important in detecting tiny force or mass [1-4]. Depending on the nature of the input stimuli, microcantilever sensor can be categorized as physical, chemical, or biological sensor [3]. The adsorbed analytes on a microcantilever surface cause the three changes: increase of mass, damping and stiffness changes [3,4]. These changes can result in the deflection and resonant frequency shifts of a microcantilever, which are also the mechanisms used for sensing. The reason for the increase of mass is rather straightforward. Damping, or say, the energy dissipation mechanism of a micro/nanocantilever is a complex one. Although various models and mechanisms are proposed [3,5], a clear picture still remains elusive [5]. The stiffness change is due to surface stress, which is associated with the changes of Gibbs free energy during adsorption process [3]. That the change of Gibbs surface free energy results in a surface stress is given in the Shuttleworth equation [6,7]. Dareing and Thundat [7] presented a model to show how the arrangements of adsorbed atoms on a microcantilever surface change the Lennard-Jones ( $\mathrm{LJ}$ ) potential, which essentially is the Gibbs surface free energy. Part of the LJ potential is transferred into the elastic energy, which bends the microcantilever [7]. There are two operation modes of a microcantilever

\footnotetext{
* Tel: +8610 82543970 .

E-mail address: zhangyin@Inm.imech.ac.cn
}

sensor: static mode and dynamic mode $[3,4,8]$. The static mode is used to measure surface stress via the following Stoney formula $[1-4,8]$

$\Delta \sigma=\frac{E h^{2}}{6 R(1-v)}$

where $E, v$ and $h$ are the microcantilever Young's modulus, Poisson's ratio and thickness, respectively. $R=2 L^{2} /(3 \Delta z)$ is the radius of curvature ( $L$ and $\Delta z$ are the microcantilever length and deflection at the free end). $\Delta \sigma$ is the differential surface stress, which has the unit of $\mathrm{Nm}^{-1}$ rather than pascal. Here we emphasize the word differential. Only when surface stress is differential, can a microcantilever bend. To maximize the differential surface stress, one essential strategy is used: to coat one surface of microcantilever with a layer that reacts with the analytes, whereas others are uncoated or coated with a layer inert to the analytes $[1,2,9,10]$. One prominent drawback of Eq. (1) should be pointed out here: the Stoney formula models the surface stress effect as if it is a concentrated moment acting on the free end of cantilever [11]. In other words, if the surface stress effect is described by the Stoney formula, the stiffness of microcantilever will not change [11-13], which is in contradiction with the experimental observations $[3,4,8,14]$. Furthermore, the Stoney formula cannot be used to measure the non-differential surface stress of a nanowire induced by the formation of a symmetric surface layer [14,15]. Because surface stress is proportional to the number of analytes adsorbed [1], the analyte concentration or its adsorbed mass on the microcantilever surface 
also needs to be closely monitored/controlled to realize a biomolecular recognition $[1,2]$. The static mode cannot detect the adsorbed mass.

To detect the adsorbed mass, the dynamic mode must be used, which in essence measures the resonant frequency change given as follows for a one degree of freedom (DOF) system [16]

$f^{\prime}=\frac{1}{2 \pi} \sqrt{\frac{K+\Delta K}{M+\Delta M}} \sqrt{1-\frac{(C+\Delta C)^{2}}{4 M K}}$,

$f^{\prime}$ is the resonant frequency after adsorption. $K, M$ and $C$ are the effective spring stiffness, mass and damping of a cantilever, respectively. $\Delta K, \Delta M$ and $\Delta C$ are those corresponding changes due to adsorption. As the microcantilever motion is recorded in an experiment, $C+\Delta C$ can be easily determined from the frequency response curve by the half-power method [16]. The problem is how to determine $\Delta K$ and $\Delta M . \Delta M$ can only be positive, which always reduces the resonant frequency. However, surface stress can be either tensile or compressive [1,2], which can either increases or decreases the spring stiffness $[11,12]$ and thus resonant frequency. Therefore, there are infinite combinations of $\Delta K$ and $\Delta M$ which can result in a same $f$ as indicated in Eq. (2). One strategy is to find out either $\Delta K$ or $\Delta M$ first by a different measurement method and then use the dynamic mode to find the other. For example, by measuring the concentration of adsorbed analyte ( $\Delta M$ is thus found), $\Delta K$ can then be found from Eq. (2) [17]; or by localizing the adsorption areas at the terminal end of cantilever to minimize the surface stress effect on the spring stiffness, $\Delta K=0$ can be assumed $[8,10]$, then $\Delta M$ can then be found by applying Eq. (2). Obviously, the drawback of this strategy is that extra efforts on device and design, which is not trivial at all, are needed. Another strategy is to use the static mode to measure surface stress to find out $\Delta K$ first (there are other models as discussed later, which can model the influence of surface stress on the microcantilever stiffness) and then use the dynamic mode to find out $\Delta M[8,18]$. While, the problem of this strategy is that adsorption is a dynamic process. The adsorption-induced mass and surface stress vary not only with time $[8,9]$ but also with how they are measured [9]. In chemical sensors, adsorption and desorption of gas molecules often occur at the same time [19]; in biological sensor, the receptor-ligand such as biotin-streptavidin and biotin-avidin also experiences a dynamic process of bonding-debonding due to the competition between the barrier of mechanical energy, dissociation kinetics and effect of thermal activation [20]. One vivid example on the difference of the static and dynamic modes is that the adsorption of water on the microcantilever coating layer of polymethylmethacrylate (PMMA) saturates in the static mode, whereas no saturation is observed in the dynamic mode [9]. The reason is that in the dynamic mode, the diffusion of water within the polymer and the dissolution of the polymer occur simultaneously [9]. In other words, the static mode and dynamic mode may not measure the same adsorption-induced mass and surface stress.

Mathematically speaking, using $f$ to find out $\Delta K$ and $\Delta M$ is an inverse problem. In this study, a novel method is presented to solve this inverse problem. As for a cantilever which is a continuous system with infinite resonant frequencies, the adsorption-induced mass and surface stress have different impacts on its resonant frequencies. This is the physical mechanism used to solve the inverse problem. The new method only needs the dynamic mode and two resonant frequencies to uniquely determine the adsorption-induced mass and surface stress. The new method is actually simpler to detect non-differential surface stress, which cannot be done by the static mode. The new method is also demonstrated to be very accurate for a typical adsorption scenario.

\section{Model development}

Fig. 1(a) is a schematic of a cantilever with a uniform layer of adsorption on its upper surface. As mentioned above, the Stoney formula is to model the surface stress effect as a concentrated moment applied at the beam free end [11], which has no impact on the beam stiffness and thus its resonant frequencies. The equation of motion based on the Stoney formula is thus not presented here. Fig. 1(b) is a schematic of the concentrated load modeling, in which a concentrated load $F$ and a concentrated moment $M_{b}$ are applied at the cantilever free end. For brevity, the governing equation of the concentrated load modeling and the boundary conditions are given as follows $[11,21,22]$

$(m+\Delta m) \frac{\partial^{2} w}{\partial t^{2}}+c \frac{\partial w}{\partial t}+E^{*} I \frac{\partial^{4} w}{\partial x^{4}}-F \frac{\partial^{2} w}{\partial x^{2}}=0$.

where $m$ is the beam mass per unit length and $m=\rho A$ ( $\rho$ and $A$ are the mass density and cross-section area of the beam, respectively). $\Delta m$ is the adsorption mass per unit length, which is assumed to have a uniform distribution all over the cantilever and is thus treated as a constant. In the applications of chemical and biological sensors, an adsorbed mass spanning the entire cantilever surface is preferred instead of an adsorbed one at a selected area [23], which prevents the need for selective activation of surface and avoids unspecific binding. $w=w(x, t)$ is the beam deflection and $c$ is damping. The concentrated load $F=\sigma b(\sigma$ is the adsorption induced surface stress and $b$ is the beam width) [11]. $E^{*}$ is the beam effective Young's modulus. Physically, the in-plane surface stress is in two directions, which are the $x$ and $y$ directions as shown in the coordinate system of Fig. 1 (b). $E^{*}=E /(1-v)$ is to account this biaxial loading scenario [11,24] ( $E$ and $v$ are the beam Young's modulus and Poisson's ratio, respectively); if a beam is (assumed) to bend in a cylindrical shape [25], $E^{*}=E /\left(1-v^{2}\right)[7,11]$; or simply $E^{*}=E[11,14,15] . I$ is the area moment of inertia and $I=b h^{3} / 12(h$ is the beam thickness) for a uniform rectangular beam. However, in many biosensors, different materials are coated on the microcantilever surfaces and the adsorption layer may also have influence on the microcantilever bending stiffness $[1,4]$. The microcantilever in general should be treated as a composite with mutilayers [4] and its bending stiffness is often calibrated by the resonance method [18].

The boundary conditions of a cantilever beam under a concentrated load and concentrated moment at its free end are the followings $[11,21,22]$

$$
\begin{aligned}
w(0, t)= & 0, \frac{\partial w}{\partial x}(0, t)=0, E^{*} I \frac{\partial^{2} w}{\partial x^{2}}(L, t)=M_{b}, E^{*} I \frac{\partial^{3} w}{\partial x^{3}}(L, t) \\
& -F \frac{\partial w}{\partial x}(L, t)=0 .
\end{aligned}
$$

where $M_{b}=\sigma b h / 2$ is the concentrated moment and $L$ is the beam length. It is noticed that the concentrated moment does not appear in the governing equation.

The distributed load modeling as shown in Fig. 1(c) gives the following governing equation [11]

$(m+\Delta m) \frac{\partial^{2} w}{\partial t^{2}}+c \frac{\partial w}{\partial t}+E^{*} I \frac{\partial^{4} w}{\partial x^{4}}-s(L-x) \frac{\partial^{2} w}{\partial x^{2}}+s \frac{\partial w}{\partial x}=0$.

where $s=F / L=\sigma b / L$ is the uniformly distributed load. Physically Eq. (5) describes surface stress as a uniformly distributed axial load along the length direction [11]. In comparison, Eq. (3) describes surface stress as a concentrated load $F$ applied at the cantilever free end, which, as discussed later, raises the issue of violating the 
(a)

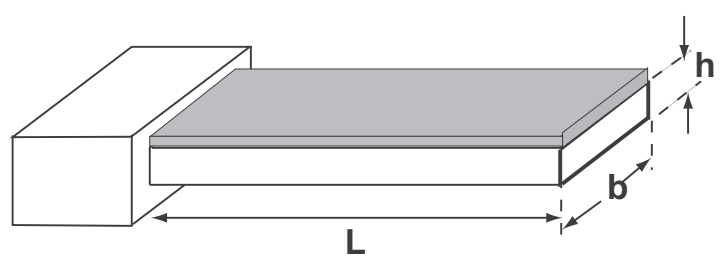

(b)
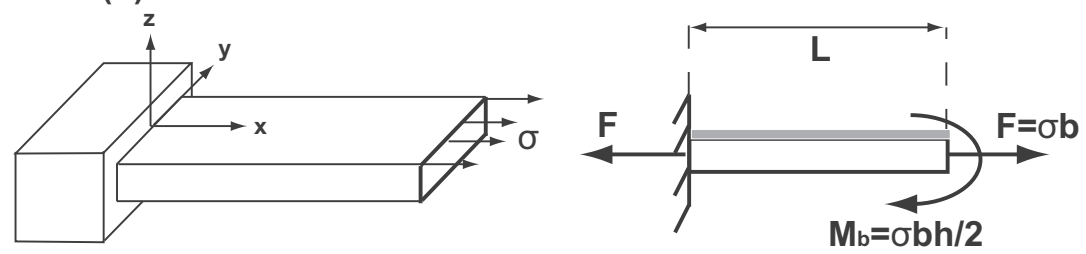

(c)
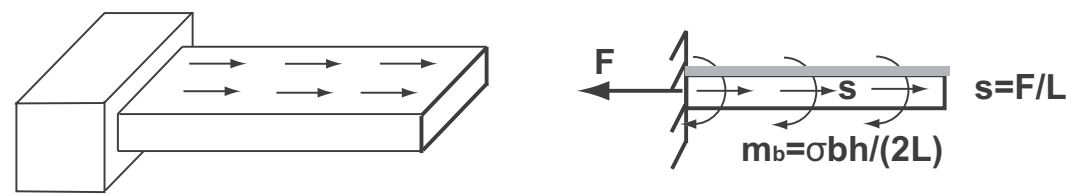

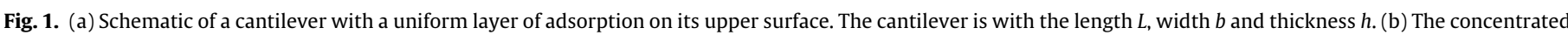

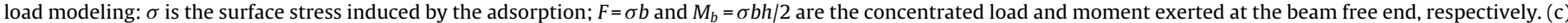
The distributed load modeling: $s=F / L=\sigma b / L$ and $m_{b}=\sigma b h /(2 L)$ are the uniformly distributed load and moment along the length direction, respectively.

boundary conditions. The corresponding boundary conditions for the distributed load model are as follows [11]

$w(0, t)=0, \frac{\partial w}{\partial x}(0, t)=0, \frac{\partial^{2} w}{\partial x^{2}}(L, t)=0, E^{*} I \frac{\partial^{3} w}{\partial x^{3}}(L, t)+m_{b}=0$.

where $m_{b}=\sigma b h /(2 L)$ is the uniformly distributed bending moment.

Because the purpose here is to find the resonant frequencies, the presence of concentrated moment $M_{b}$ in the boundary conditions of Eq. (4) and distributed moment $m_{b}$ in those of Eq. (6) causes a problem of formulating an eigenvalue problem. To solve the problem, the beam deflection is expressed as the following two parts:

$w(x, t)=w_{o}(x)+w_{1}(x, t)$.

where $w_{o}(x)$ is the equilibrium, which is independent of time $t$. $w_{1}(x, t)$ is the displacement away from the equilibrium. With the presence of differential surface stress, the cantilever bends away from the horizontal position. The reason of breaking the deflection into the above two parts can be answered by the following analogy: for one DOF spring-mass system, whether it is placed horizontally, or vertically or on a slope only changes the equilibrium position and has no impact on the system resonant frequency, which is only determined by the spring stiffness and mass. For the concentrated load modeling, $w_{0}(x)$ satisfies boundary conditions of Eq. (4) and equilibrium equation of $E^{*} I\left(\partial^{4} w_{o} / \partial x^{4}\right)-F\left(\partial^{2} w_{o} / \partial x^{2}\right)=0$; for the distributed load modeling, $w_{0}(x)$ satisfies boundary conditions of Eq. (6) and equilibrium equation of $E^{*} I\left(\partial^{4} w_{o} / \partial x^{4}\right)-s(L-$ $x)\left(\partial^{2} w_{0} / \partial x^{2}\right)+s\left(\partial w_{0} / \partial x\right)=0$. Substitute Eq. (7) into Eqs. (3) and
(4), the following governing equation and boundary conditions are derived for the concentrated load modeling

$$
\begin{gathered}
(m+\Delta m) \frac{\partial^{2} w_{1}}{\partial t^{2}}+c \frac{\partial w_{1}}{\partial t}+E^{*} I \frac{\partial^{4} w_{1}}{\partial x^{4}}-F \frac{\partial^{2} w_{1}}{\partial x^{2}}=0 \\
w_{1}(0, t) 0=0, \frac{\partial w_{1}}{\partial x}(0, t)=0, E^{*} I \frac{\partial^{2} w_{1}}{\partial x^{2}}(L, t)=0, E^{*} I \frac{\partial^{3} w_{1}}{\partial x^{3}}(L, t) \\
-F \frac{\partial w_{1}}{\partial x}(L, t)=0
\end{gathered}
$$

Clearly, only the boundary conditions change, which serves the purpose of formulating the eigenvalue problem as shown later. Actually Eqs. (8) and (9) are also the governing equation and boundary conditions for the micro/nanostructure with the symmetric/non-differential surface stress [11,21,22].

Similarly, the following governing equation and boundary conditions are derived for the distributed load modeling

$(m+\Delta m) \frac{\partial^{2} w_{1}}{\partial t^{2}}+c \frac{\partial w_{1}}{\partial t}+E^{*} I \frac{\partial^{4} w_{1}}{\partial x^{4}}-s(L-x) \frac{\partial^{2} w_{1}}{\partial x^{2}}+s \frac{\partial w_{1}}{\partial x}=0$.

$w_{1}(0, t)=0, \frac{\partial w_{1}}{\partial x}(0, t)=0, \frac{\partial^{2} w_{1}}{\partial x^{2}}(L, t)=0, E^{*} I \frac{\partial^{3} w_{1}}{\partial x^{3}}(L, t)=0$.

Again, the governing equation does not change and boundary conditions change. Because adsorption is assumed to occur only on the cantilever upper surface, surface stress is differential, which is also responsible for generating bending moment. For the 
non-differential surface stress loading scenario [14,15], the steps starting from Eq. (7) to Eq. (11) are not needed.

By introducing $\xi=x / L, W_{1}=w_{1} / L$ and $\tau=\sqrt{E^{*} I /\left(m L^{4}\right)} t$ (here $\sqrt{E^{*} I /\left(m L^{4}\right)}$ is with the unit of Hertz and it is the same order of the first natural frequency of a uniform and undamped cantilever beam $[26,27])$, the governing equation of the concentrated load modeling, Eq. (8), is nondimensionalized as follows

$(1+\alpha) \frac{\partial^{2} W_{1}}{\partial \tau^{2}}+C \frac{\partial W_{1}}{\partial \tau}+\frac{\partial^{4} W_{1}}{\partial \xi^{4}}-\Lambda \frac{\partial^{2} W_{1}}{\partial \xi^{2}}=0$.

where the dimensionless quantities $\alpha, C$ and $\Lambda$ are defined as follows

$\alpha=\frac{\Delta m}{m}, C=c \sqrt{\frac{L^{4}}{E^{*} I m}}, \Lambda=\frac{\sigma b L^{2}}{E^{*} I}$

Clearly $\alpha$ indicates the ratio of the adsorption mass to the beam mass and $\Lambda$ indicates the ratios of the surface stress to the beam stiffness per unit width. $C$ is the dimensionless damping. The boundary conditions of Eq. (9) is now nondimensionalized as follows

$$
\begin{aligned}
W_{1}(0, \tau)= & 0, \frac{\partial W_{1}}{\partial \xi}(0, \tau)=0, \frac{\partial^{2} W_{1}}{\partial \xi^{2}}(1, \tau)=0, \frac{\partial^{3} W_{1}}{\partial \xi^{3}}(1, \tau) \\
& -\Lambda \frac{\partial W_{1}}{\partial \xi}(1, \tau)=0
\end{aligned}
$$

For the distributed load modeling, the governing equation of Eq. (10) and boundary conditions of Eq. (11) are nondimensionalized as follows

$(1+\alpha) \frac{\partial^{2} W_{1}}{\partial \tau^{2}}++C \frac{\partial W_{1}}{\partial \tau}+\frac{\partial^{4} W_{1}}{\partial \xi^{4}}-\Lambda(1-\xi) \frac{\partial^{2} W_{1}}{\partial \xi^{2}}+\Lambda \frac{\partial W_{1}}{\partial \xi}=0$

and

$W_{1}(0, \tau)=0, \frac{\partial W_{1}}{\partial \xi}(0, \tau)=0, \frac{\partial^{2} W_{1}}{\partial \xi^{2}}(1, \tau)=0, \frac{\partial^{3} W_{1}}{\partial \xi^{3}}(1, \tau)=0$

To compute the eigenfrequencies of Eq. (15), the Galerkin method $[21,26,28]$ is applied, which assumes the following form for $W_{1}(\xi$, $\tau)$

$W_{1}(\xi, \tau)=\sum_{j=1}^{N} a_{j}(\tau) \phi_{j}(\xi)$

where $N$ is the mode number; $a_{j}(\tau)$ is the time-dependent modal amplitude and $\phi_{j}(\xi)$ is the jth mode shape of a uniform cantilever beam. It is noticed that the presence of (dimensionless) axial load $\Lambda$ in the boundary conditions of Eq. (14) of the concentrated load modeling can have significant impact on the shape of $\phi_{j}(\xi)[21,28]$, which as a result can also influence the accuracy of resonant frequencies. The detailed procedures of finding $\phi_{j}(\xi)$ under different axial loadings are presented in reference [28]. Here it is necessary for us to have a brief discussion and comparison on these two modelings. The free end of a cantilever under a surface stress loading is free from a net force [24]. The presence of an axial load at the free end as reflected in the fourth boundary condition of Eq. (14) is therefore, according to Lachut and Sader [24], "in direct violation of Newton's third law". In comparison, the distributed load modeling indeed guarantees the zero net force of the cantilever free end. It is also clear from Eqs. (12) and (15) that the influence of surface stress on the resonant frequencies acts as an axial load. Besides adsorption, the processes such as electron beam induced deposition (EBID) [29] and low pressure chemical vapor deposition (LPCVD) [30], which are frequently used in surface micromaching, microlithography and manufacturing of micro/nanomechanical devices, can also induce the changes of mass, residual stress and its gradients. The above modelings can also apply to these scenarios.

Substitute Eq. (17) into Eq. (12), time $\phi_{i}^{0}(\xi)$ and integrate from 0 to 1 , the following equation is obtained

$\mathrm{M}^{\mathrm{I}} \mathrm{q}+\mathrm{D}^{\mathrm{I}} \dot{\mathrm{q}}+\mathrm{K}^{\mathrm{I}} \mathrm{q}=\mathbf{0}$.

Here $\dot{(})=\frac{\partial}{\partial \tau}$ and $\mathbf{q}$ is a vector given as $\mathbf{q}=\left(a_{1}, a_{2}, \ldots, a_{N}\right)^{T} . \mathbf{M}^{I}$, $\mathbf{D}^{I}$ and $\mathbf{K}^{I}$ are the $N \times N$ matrices of mass, damping and stiffness, respectively. Matrices $\mathbf{M}^{I}, \mathbf{D}^{I}$ and $\mathbf{K}^{I}$ are derived by using the orthonormality property of $\phi_{j}(\xi)[26]$ as follows

$\mathrm{M}_{\mathrm{ij}}^{\mathrm{I}}= \begin{cases}(1+\alpha) \int_{0}^{1} \phi_{i}^{2}(\xi) \mathrm{d} \xi, & \mathrm{i}=\mathrm{j} \\ 0, & \mathrm{i} \neq \mathrm{j}\end{cases}$

$D_{i j}^{I}= \begin{cases}C \int_{0}^{1} \phi_{i}^{2}(\xi) d \xi, & i=j \\ 0, & i \neq j,\end{cases}$

and

$\mathrm{K}_{\mathrm{ij}}^{\mathrm{I}}=\int_{0}^{1} \phi_{i}(\xi) \frac{\partial^{4} \phi_{j}(\xi)}{\partial \xi^{4}} \mathrm{~d} \xi-\Lambda \int_{0}^{1} \phi_{i}(\xi) \frac{\partial^{2} \phi_{j}(\xi)}{\partial \xi^{2}} \mathrm{~d} \xi$.

Similarly, for the distributed load modeling, the following equation is obtained

$\mathrm{M}^{\mathrm{II}} \mathrm{q}+\mathrm{D}^{\mathrm{II}} \dot{\mathrm{q}}+\mathrm{K}^{\mathrm{II}} \mathrm{q}=\mathbf{0}$.

where $\mathbf{M}^{I I}=\mathbf{M}^{I}$ and $\mathbf{D}^{I I}=\mathbf{D}^{I}$. Now the stiffness matrix $\mathbf{K}^{I I}$ is given as follows

$$
\begin{aligned}
\mathrm{K}_{\mathrm{ij}}^{\mathrm{II}}= & \int_{0}^{1} \phi_{i}(\xi) \frac{\partial^{4} \phi_{j}(\xi)}{\partial \xi^{4}} \mathrm{~d} \xi-\Lambda \int_{0}^{1}(1-\xi) \phi_{i}(\xi) \frac{\partial^{2} \phi_{j}(\xi)}{\partial \xi^{2}} \mathrm{~d} \xi \\
& +\Lambda \int_{0}^{1} \phi_{i}(\xi) \frac{\partial \phi_{j}(\xi)}{\partial \xi} \mathrm{d} \xi
\end{aligned}
$$

Because of the presence of damping, Eqs. (18) and (22) actually do not formulate the exact eigenvalue problem for us to find out the resonant frequencies. Both Eqs. (18) and (22) are the damped nongyroscopic system [31]. For the concentrated load modeling, the eigenvalue problem is formulated by manipulating Eq. (18) as follows [31]

$\mathrm{M}^{*} \dot{\mathrm{Y}}+\mathrm{K}^{*} \mathrm{Y}=\mathbf{0}$.

where $\mathbf{Y}=(\dot{\mathrm{q}}, \mathbf{q})$ is a $2 N$ vector; $\mathbf{M}^{*}$ and $\mathbf{K}^{*}$ are the $2 N \times 2 N$ matrices defined as follows [31]

$\mathrm{M}^{*}=\left(\begin{array}{cc}\mathrm{M}^{\mathrm{I}} & \mathbf{0} \\ \mathbf{0} & -\mathrm{K}^{\mathrm{I}}\end{array}\right), \mathrm{K}^{*}=\left(\begin{array}{cc}\mathrm{D}^{\mathrm{I}} & \mathrm{K}^{\mathrm{I}} \\ \mathrm{K}^{\mathrm{I}} & \mathbf{0}\end{array}\right)$

For the distributed loading, the eigenvalue problem is formulated by substituting $\mathbf{M}^{I}$ by $\mathbf{M}^{I I}$, $\mathbf{D}^{I}$ by $\mathbf{D}^{I I}$ and $\mathbf{K}^{I}$ by $\mathbf{K}^{I I}$.

\section{Results and discussions}

For simplicity and comparison reasons, damping is set as zero (i.e., $C=0$ ) in all computations presented here, which is also the case in references $[14,22,24]$. The high quality factor (i.e., small damping) is a much sought-after property in many applications 


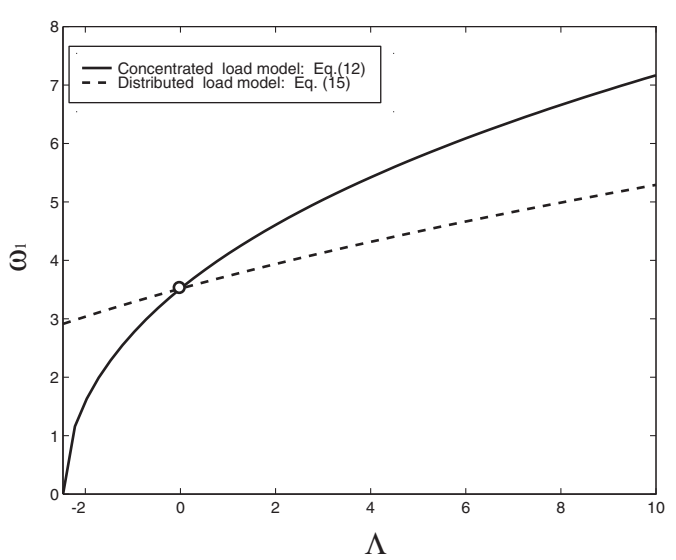

Fig. 2. The first resonance frequency $\left(\omega_{1}\right)$ calculated by the concentrated load modeling and the distributed modeling as a function of $\Lambda$ when $\alpha=0$. The intersection is marked with a circle.

of micro/nano-resonator, which can significantly enhance the sensitivity [23]. Therefore, the shifts of resonant frequencies due to small damping can be ignored in many dynamic modelings of the microstructure vibration in air or vacuum. The presence of damping reduces the resonant frequencies and the eigenfrequency computation of a damped system using the above Galerkin method can be found in reference [26]. As mentioned above, damping is usually determined by the so-called half-power method from the frequency response curve obtained by experiment [16]. It also needs to keep in mind that damping varies when a microbeam vibrates at different resonances [23]. The mode number is taken as $N=4$ in all the resonant frequency computations.

Fig. 2 plots the first eigenfrequency $\left(\omega_{1}\right)$ calculated by the concentrated load model of Eq. (18) and the distributed load model of Eq. (22) as the function of $\Lambda$ when $\alpha=0$. The first two resonant frequencies/eigenfrequencies with $\alpha=\Lambda=0$ are given as follows [27]

$\omega_{1}^{0}=1.875104^{2}=3.516015, \omega_{2}^{0}=4.694091^{2}=22.034492$.

Because surface stress here is modeled to have the axial load effect and a tensile/compressive axial load stiffens/softens a structure, the eigenfrequencies calculated by both models change with $\Lambda$. Large compressive surface stress can lead to the buckling of a microcantilever beam [32] because of the softening effect of a compressive axial load. The effective stiffness of a structure becomes zero at the buckling load [33], which causes the first eigenfrequency to be zero. When the buckling load of $\Lambda=-\pi^{2} / 4 \approx-2.46$ is reached [33], $\omega_{1}$ of the concentrated load model becomes zero as shown in Fig. 2. However, $\omega_{1}$ of the distributed load model is not zero. Clearly $\Lambda$ in the distributed load model has much less impact on the eigenfrequency. The reason has already been given by Timoshenko that a cantilever under a distributed load of $s=F / L$, its effect on $\omega_{1}$ is as if a concentrated load of $7 F / 20$ applied at the free end [34]. Clearly these two models have large difference on the stiffening and softening effects induced by surface stress. The two curves have only one intersection, which corresponds to $\left(\Lambda, \omega_{1}\right)=\left(0, \omega_{1}^{0}\right)$ and is marked as a circle.

In the following figures, only the distributed load model is used to show how to use the shifts of eigenfrequencies to determine the adsorbed mass and surface stress. Physically $\alpha=\Lambda=0$ corresponds to the no adsorption case, which can also be used to calibrate a beam $[18,36]$. As mentioned above, in order to realize certain function or to enlarge sensitivity, the microcantilever is usually coated with several layers of different thicknesses and materials $[1,4]$, which makes it very difficult to specify the beam bending stiffness of $E^{*} I$ and mass per unit length of $m$ [35]. Even for a given material, for example, silicon nitride $\left(\mathrm{SiN}_{x}\right)$, which is commonly used to fabricate a microcantilever [1], its Young's modulus varies from 130 to $385 \mathrm{GPa}$ and its Poisson ratio ranges from 0.2 to 0.3 because the exact atomic ratio between $\mathrm{Si}$ and $\mathrm{N}$ is not determined [35]. Furthermore, at the small scale, surface elasticity can also change the effective Young's modulus of a microcantilever [6]. The dimensional fundamental resonant frequency, $f_{1}^{0}$ is related with the above dimensionless resonant frequency as $f_{1}^{0}=\omega_{1}^{0} \sqrt{E^{*} I /\left(m L^{4}\right)} / 2 \pi$. Once $f_{1}^{0}$ is measured, $E^{*} I / m$ can be found as $E^{*} I / m=\left(2 \pi f_{1}^{0} L^{2} / \omega_{1}^{0}\right)^{2}$. The detailed procedures of using the resonant frequency to calibrate a microcantilever with and without the damping effect can be found in references $[18,36]$.

For a microcantilever sensor made from silicon or silicon nitride, $E^{*} \sim 10^{11} \mathrm{Nm}^{-2}, h \sim 1 \mu \mathrm{m}, L \sim 100 \mu \mathrm{m}, \rho \sim 1.6 \times 10^{3} \mathrm{~kg} \mathrm{~m}^{-3}$; the surface stress induced by DNA adsorption on a self-assembled monolayer(SAM) is around $0.02 \sim 1.5 \mathrm{Nm}^{-1}$ [4]. Therefore, $\Lambda$ can be calculated from Eq. (13). Here $\alpha=10^{-3}$ and $\Lambda=10^{-2}$ are set, which are typical values in the above microcantilever application scenarios. Substitute these two $\Lambda$ and $\alpha$ values into Eq. (22), the first two eigenfrequencies are obtained as follows

$\omega_{1}=3.516491, \omega_{2}=22.025444$.

The (dimensionless) adsorption mass $(\alpha)$ can only be positive, which always softens the beam, i.e., decreases the eigenfrequencies. The (dimensionless) surface stress $(\Lambda)$ can be either positive or negative. The positive $\Lambda$, which is tensile, stiffens the beam and the negative compressive $\Lambda$ softens the beam. Compared with the eigenfrequencies of $\alpha=\Lambda=0$ as given in Eq. (26), it is interesting to notice that $\omega_{1}$ increases, whereas $\omega_{2}$ decreases. Physically the reason is that the adsorbed mass and surface stress have different impact on the eigenfrequencies and this is the exact mechanism for us to determine adsorption-induced mass and surface stress. It is noticed that the eigenfrequency shifts due to these two tiny parameters of $\Lambda$ and $\alpha$ are also very tiny, which is the typical application scenarios of many resonator sensors. Because the eigenfrequency of a beam is proportional to $\sqrt{E I /\left(m L^{4}\right)} \propto h / L^{2} \times \sqrt{E / \rho}$ [26], two major strategies of enhancing the sensitivity of detecting the eigenfrequency shifts are: (1) to scale down the structure size [37-39], which makes factor of $h / L^{2}$ larger; (2) to use the materials with large $E / \rho$, such as graphene [40] and carbon nanotube [41]. Both result in higher eigenfrequencies. Therefore, a small fractional change in eigenfrequency is still absolutely large enough to be detected [42]. By using these two strategies, the giga-Hertz resonator has been fabricated [37] and the capability of detecting (dimensionless) adsorption mass as small as $\alpha=6.6 \times 10^{-5}$ has been achieved [41].

In the real sensor application, $\Lambda$ and $\alpha$ are unknown; the eigenfrequencies are extracted from the beam frequency response curves $[8,14,16]$. Therefore, using the eigenfrequencies (shifts) to determine $\Lambda$ and $\alpha$ forms an inverse problem. Fig. 3 plots the variation of the first eigenfrequency, $\omega_{1}$, as a function of $\Lambda$ and $\alpha$, which is a titled plane. $\omega_{1}$ increases monotonically with the increase of $\Lambda$ and decreases monotonically with the increase of $\alpha$. The level plane is the one with the fixed first eigenfrequency value of $\omega_{1}=3.516491$. The intersection of these two planes is all the combinations of $\Lambda$ and $\alpha$, which result in the same first eigenfrequency of $\omega_{1}=3.516491$. The intersection is a line marked in Fig. 3. This line also indicate that the combinations resulting in a same eigenfrequency are infinite. Fig. 4 plots the second eigenfrequency of $\omega_{2}$ as a function of $\Lambda$ and $\alpha$. The level plane is the one with the fixed value of $\omega_{2}=22.025444$. Again, the intersection line of the two planes indicates the combinations of $\Lambda$ and $\alpha$ resulting in $\omega_{2}=24.628$. Clearly, for any given values of $\Lambda$ and $\alpha$, each eigenfrequency is uniquely determined by Eq. (22). As an inverse problem, there are infinite combinations of $\Lambda$ and $\alpha$ for a given eigenfrequency. However, as shown in Fig. 5, 


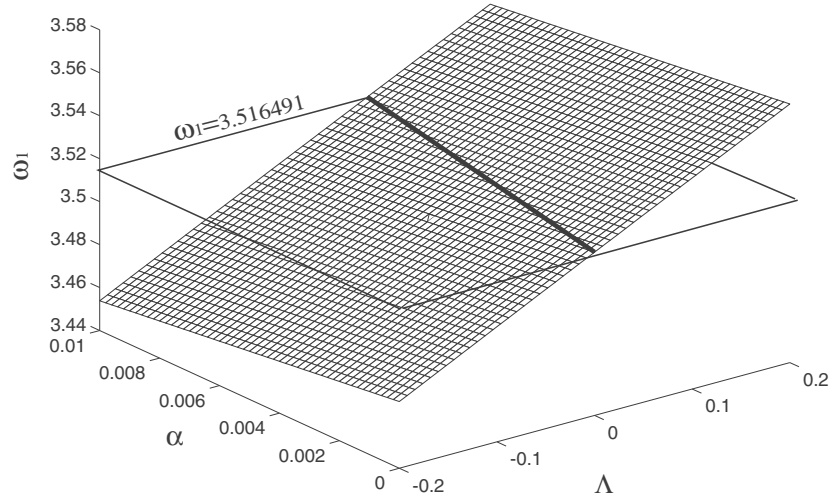

Fig. 3. Variation of the first resonance frequency $\left(\omega_{1}\right)$ as a function of $\alpha$ and $\Lambda$. The level plane is the one with a fixed resonance frequency of $\omega_{1}=3.516491$. The intersection of the two planes is marked with a solid line.

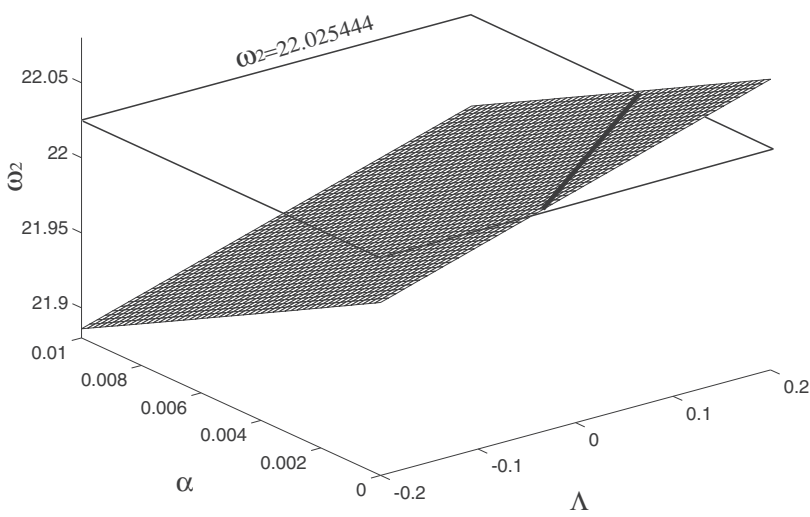

Fig. 4. Variation of the second resonance frequency $\left(\omega_{2}\right)$ as a function of $\alpha$ and $\Lambda$. The level plane is the one with a fixed resonance frequency of $\omega_{2}=22.025444$. The intersection of the two planes is marked with a solid line.

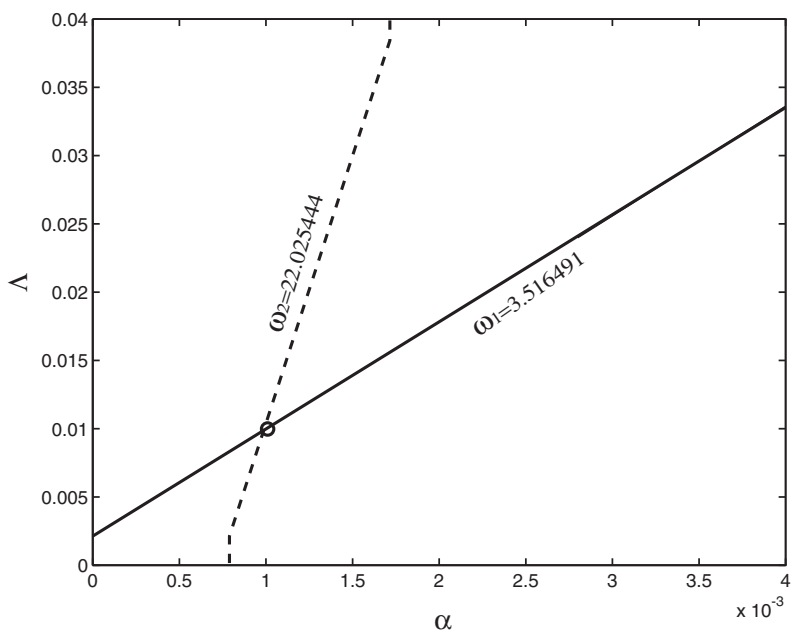

Fig. 5. The combinations of $\alpha$ and $\Lambda$ for the resonant frequencies of $\omega_{1}=3.516491$ and $\omega_{2}=22.025444$, which are two lines marked in Figs. 3 and 4 . The intersection is marked with a circle, which corresponds to $(\alpha, \Lambda)=\left(10^{-3}, 10^{-2}\right)$.

for two given eigenfrequencies, their combinations of $\Lambda$ and $\alpha$, which are two lines, intersect. Physically, this intersection is used to uniquely determine the combination of $\alpha$ and $\Lambda$. In Fig. 5, the intersection of the combinations of $\alpha$ and $\Lambda$ for $\omega_{1}=3.516491$ and $\omega_{2}=22.025444$ is marked as a circle, which happens to be exactly $(\alpha, \Lambda)=\left(10^{-3}, 10^{-2}\right)$.

\section{Conclusion}

Adsorption-induced mass and surface stress play different roles in the eigenfrequency shifts of a cantilever. There are infinite combinations of the mass and surface stress which can result in the same shift of one resonant frequency. However, there is only one combination which can result in the same shifts of two resonant frequencies, which is utilized in this study as an effective mechanism to solve the inverse problem of using the resonant frequency shifts to determine the mass and surface stress. In many sensor applications, adsorption-induced surface stress and mass are separately determined by static and dynamic modes. In some scenarios when surface stress is not differential, the static mode cannot determine surface stress. With the method presented here, the static mode is unnecessary. Two different models are presented. Because of their different modelings on the impact of surface stress, the eigenfrequencies calculated by these two models are significantly different. As the concentrated load model may violate the boundary conditions of a cantilever under a surface stress loading, only the distributed load model is used for solving the inverse problem. However, the method presented in this study, which in essence is to use two resonant frequency shifts to determine the adsorbed mass and surface stress, can also be applied to the concentrated load model. The method can be easily extended to the sensors with the clamped-clamped boundary conditions (whose purpose is to achieve larger eigenfrequencies) [37] by simply changing the mode shape of $\phi_{j}$. The first and second resonant frequencies are used in this study to solve the inverse problem. When a microcantilever vibrates at a higher resonant frequency, its sensitivity can have the improvement of orders of magnitude due to the significant decrease of damping [23]. This method can also be easily extended to this application scenario by simply calculating the other two different resonant frequencies.

\section{Acknowledgments}

The research has been supported by the National Natural Science Foundation of China (NSFC No. 110212622 and 11023001) and Chinese Academy of Sciences (Grant no. KJCX2-EW-L03).

\section{References}

[1] R. Berger, E. Delamarche, H.P. Lang, Ch. Gerber, J.K. Gimzewski, E. Meyer, H.J Güntherodt, Surface stress in self-assembly of alkanethiols on gold, Science 276 (1997) 2021-2024.

[2] J. Fritz, M.K. Baller, H.P. Lang, H. Rothuizen, P. Vettiger, E. Meyer, H.J. Güntherodt, Ch. Gerber, J.K. Gimzewski, Translating biomolecular recognition into nanomechanics, Science 288 (2000) 316-318.

[3] N.V. Lavrik, M.J. Sepaniak, P.G. Datskos, Cantilever transducers as a platform for chemical and biological sensors, Review of Scientific Instruments 75 (2004) 2229-2253.

[4] S.T. Koev, M.A. Powers, H. Yi, L. Wu, W.E. Bentley, G.W. Rubloff, G.F. Payne, R. Ghodssi, Mecho-transduction of DNA hybridization and dopamine oxidation through electrodeposited chitosan network, Lab on a Chip 7 (2007) 103-111.

[5] P. Mohanty, D.A. Harrington, K.L. Ekinci, Y.T. Yang, M.J. Murphy, M.L. Roukes, Intrinsic dissipation in high-frequency micromechanical resonator, Physical Review B 66 (2002) 085416.

[6] P. Müller, A. Saúl, Elastic effects on surface physics, Surface Science Reports 54 (2004) 157-258.

[7] D.W. Dareing, T. Thundat, Simulation of adsorption-induced stress of a microcantilever sensor, Journal of Applied Physics 97 (2005) 043526.

[8] G.Y. Chen, T. Thundat, E.A. Wachter, R.J. Warmack, Adsorption-induced surface stress and its effects on resonance frequency of microcantilever, Journal of Applied Physics 77 (1995) 3618-3622.

[9] F.M. Battiston, J.P. Ramseyer, H.P. Lang, M.K. Baller, Ch. Gerber, J.K. Gimzewski, E. Meyer, H.J. Güntherodt, A chemical sensor based on a microfabricated cantilever array with simultaneous resonance-frequency and bending readout, Sensors and Actuators, B 77 (2001) 122-131.

[10] T. Thundat, L. Maya, Monitoring chemical and physical changes on subnanogram quantities of platinum dioxide, Surface Science 430 (1999) L546-L552.

[11] Y.Zhang, Q. Ren, Y.P. Zhao, Modeling analysis of surface stress on a rectangular cantilever beam, Journal of Physics D-Applied Physics 37 (2004) 2140-2145. 
[12] Y. Zhang, Y.P. Zhao, Applicability range of Stoney's formula and modified formulas for a film/substrate bilayers, Journal of Applied Physics 99 (2006) 053513.

[13] P. Müller, R. Kern, About the measurement of absolute isotropic surface stress of crystals, Surface Science 301 (1994) 386-398.

[14] K.B. Gavan, H. Westra, E. van der Drift, W. Venstra, H. van der Zant, Sizedependent effective Young's modulus of silicon nitride cantilevers, Applied Physics Letters 94 (2009) 233108.

[15] J. He, C.M. Liley, Surface effect on the elastic behavior of static bending nanowires, Nano Lett. 8 (2008) 1798-1802.

[16] L. Meirovitch, Analytical Methods in Vibrations, Macmillan Publishing Co. Inc., New York, 1967.

[17] S. Cherian, T. Thundat, Determination of adsorption-induced variation in the spring constant of a microcantilever, Applied Physics Letters 80 (2002) 2219-2221.

[18] A.W. McFarland, M.A. Poggi, L.A. Bottomley, J.S. Colton, Characterization of microcantilever solely by frequency response acquisition, Journal of Micromechanics and Microengineering 15 (2005) 785-791.

[19] K.L. Ekinci, M.L. Roukes, Nanoelectromechanical systems, Review of Scientific Instruments 76 (2005) 061101.

[20] R. Merkel, P. Nassoy, A. Leung, K. Ritchie, E. Evans, Energy landscapes of receptor-ligand bonds explored with dynamic force spectroscopy, Nature 397 (1999) 50-53.

[21] J. Dorignac, A. Kalinowski, S. Erramilli, P. Mohanty, Dynamic response of nanomechanical oscillators in immiscible fluid for in vitro biomolecular recognition, Physical Review Letters 96 (2006) 186105.

[22] Q. Ren, Y.P. Zhao, Influence of surface stress on frequency of microcantileverbased biosensors, Microsystem Technologies 10 (2004) 307-314.

[23] M.K. Ghatkesar, V. Barwich, T. Braun, J. Ramseyer, Ch. Gerber, M. Hegner, H.P. Lang, U. Drechsler, M. Despont, Higher modes of vibration increase mass sensitivity in nanomechanical microcantilever, Nanotechnology 18 (2007) 445502.

[24] M.L. Lachut, J.E. Sader, Effect of surface stress on the stiffness of cantilever plates, Physical Review Letters 99 (2007) 206102.

[25] S.P. Timoshenko, S. Woinowsky-Krieger, Theory of Plates and Shells, 2nd ed., McGraw-Hill, New York, 1959.

[26] Y. Zhang, K.D. Murphy, Multi-modal analysis on the intermittent contact dynamics of atomic force microscope, Journal of Sound and Vibration 330 (2011) 5569-5582.

[27] T.C. Chang, R.R. Craig, Normal modes of uniform beams, Journal of the Engineering Mechanics 195 (1969) 1027-1031.

[28] Y. Zhang, Y.P. Zhao, Numerical and analytical study on the pull-in instability of micro-structure under electrostatic loading, Sensors and Actuators, A 127 (2006) 366-380.
[29] S. Zaitsev, O. Shtempluck, E. Buks, Effects of electron beam induced carbon deposition on the mechanical properties of a mechanical oscillator, Sensors and Actuators, A 179 (2012) 237-241.

[30] Y. Zhang, Y.P. Zhao, An effective method of determining the residual stress gradients, Microsystem Technologies 12 (2006) 357-364.

[31] L. Meirovitch, Computational Methods in Structural Dynamics, Sijthoff \& Noordhoff Inc., Rockville, MD, 1980.

[32] G.F. Wang, X.Q. Feng, Surface effects on buckling of nnaowires under uniaxial compression, Applied Physics Letters 94 (2009) 141913.

[33] A. Chajes, Principles of Structural Stability Theory, Prentice-Hall, Inc., Englewood Cliffs, NJ, 1974.

[34] S.P. Timoshenko, Vibration Problem Engineering, 2nd ed., D. van Nostrand, New York, 1937.

[35] M. Godin, V. Tabard-Cossa, P. Grütter, Quantitative surface stress measurements using a microcantilever, Applied Physics Letters 79 (2001) 551-553.

[36] J.E. Sader, J. Chon, O. Mulvaney, Calibration of rectangular atomic force microscope cantilevers, Review of Scientific Instruments 70 (1999) 3967-3969.

[37] X. Huang, C.A. Zorman, M. Mehregany, M.L. Roukes, Nanodevice motion at microwave frequencies, Nature 421 (2003) 496.

[38] K.L. Ekinci, Y.T. Yang, M.L. Roukes, Ultimate limits to inertial mass sensing based upon nanoelectromechanical systems, Journal of Applied Physics 95 (2004) 2682-2689.

[39] D. Ramos, J. Tamayo, J. Mertens, M. Calleja, A. Zaballos, Origin of the response of nanomechanical resonators to bacteria adsorption, Journal of Applied Physics 100 (2006) 106105.

[40] J.S. Bunch, A.M. van der Zande, S.S. Verbridge, I.W. Frank, D.M. Tanenbaum, J.M. Parpia, H.G. Craighead, P.L. McEuen, Electromechanical resonators from graphene sheets, Science 315 (2007) 490-493.

[41] H. Chiu, P. Hung, H. Postma, M. Bockrath, Atomic-scale mass sensing using carbon nanotube resonators, Nano Letters 8 (2008) 4342-4346.

[42] R.G. Knobel, Weighing single atoms with a nanotube, Nature Nanotechnology 3 (2008) 525-526.

\section{Biography}

Yin Zhang, BS, Department of Mechanics, Peking University, Beijing, China, 1997 and $\mathrm{PhD}$, Department of Mechanical Engineering, University of Connecticut,Storrs, Connecticut, USA, 2003. From 2003 to 2004, he worked as an assistant professor in the State Key Laboratory of Nonlinear Mechanics (LNM), Institute of Mechanics, Chinese Academy of Sciences (CAS) and as an associate professor since 2004. His current research focuses on the MEMS/NEMS structural mechanics, dynamics and interface properties. 